\title{
LOWER ESTIMATES OF TOP LYAPUNOV EXPONENT FOR COOPERATIVE RANDOM SYSTEMS OF LINEAR ODES
}

\author{
JANUSZ MIERCZYŃSKI
}

(Communicated by Yingfei Yi)

\begin{abstract}
For cooperative random linear systems of ordinary differential equations a method is presented of obtaining lower estimates of the top Lyapunov exponent. The proofs are based on applying some polynomial Lyapunovlike function. Known estimates for the dominant eigenvalue of a nonnegative matrix due to G. Frobenius and L. Yu. Kolotilina are shown to be specializations of our results.
\end{abstract}

In many dynamical systems (DSs) stemming from population dynamics persistence is an important concept: mathematically speaking, some (usually invariant, or at least forward invariant) subset $Y$ of the phase space $X$ should have a property that (forward semi)trajectories of points close to $Y$ have their $\omega$-limit sets disjoint from $Y$, or that (forward semi)trajectories of points close to $Y$ eventually leave some preassigned neighborhood of $Y$ (that stronger property is usually called permanence).

$Y$ corresponds to some constituents of the population being absent, therefore it has a structure of a $C^{1}$ submanifold, or a (forward) invariant family of $C^{1}$ submanifolds. When the dynamical system in question is smooth enough, its linearization leaves the tangent bundle of $Y$ invariant. Consequently one can legitimately speak of normal Lyapunov exponents, that is, exponential growth rates of those trajectories of the linearization at $Y$ which correspond to a subbundle complementary to the tangent bundle of $Y$. It is to be expected that positivity of such normal Lyapunov exponents should imply persistence.

Indeed, there are a wealth of papers where the above implication is shown, for instance [20, 13, 6] for autonomous systems of ordinary differential equations, [17], 15] for nonautonomous systems of reaction-diffusion equations of Kolmogorov type, to mention but a few.

Even in the case when the original DS is generated by an autonomous system of ordinary differential equations (ODEs), if there are nontrivial ergodic invariant measures on $Y$ investigation of normal Lyapunov exponents requires considering time-dependent systems of linear ODEs.

Received by the editors May 25, 2013.

2010 Mathematics Subject Classification. Primary 34C12, 34D08, 37C65. Secondary 15B48, $92 \mathrm{D} 25$.

The author was supported by project S20058/I-18. 
It is straightforward then that being able to obtain estimates from below of Lyapunov exponents for time-dependent systems of linear ODEs is of great importance in the theory of persistence. Sometimes, especially in systems of ODEs encountered in animal ecology, $Y$ is a submanifold of codimension one (or the problem can be reduced to that case), so that one needs only to find sufficient conditions for the least Lyapunov exponent of a (one-dimensional) linear ODE to be positive, which is a relatively easy task.

In applications of systems of ODEs to epidemiology, the codimension of $Y$ may be greater than one. Also, an important property is that the matrix of the restriction of the linearization to a complementary subbundle of $Y$ has off-diagonal entries nonnegative (see [10], 19] and references contained therein).

Thanks to the efforts of many generations of mathematicians, numerous results are known on estimates of the dominant eigenvalue of a matrix with nonnegative off-diagonal entries, see [18, Chapter II].

One should bear in mind, however, that for many-dimensional systems $x^{\prime}=$ $A(t) x$ of linear ODEs there is no straightforward relation between the real parts of the eigenvalues of $A(t)$ and exponential growth rates of solutions of the system (see an excellent paper [8]). So, results alluded to in the previous paragraph are of limited (if any) value as regards finding estimates in the time-dependent case.

Our goal in the present paper to give easily checkable lower estimates on maximum exponential growth rates of solutions of random linear ODE systems whose matrices have nonnegative off-diagonal entries.

\section{Preliminaries}

We denote by $\mathbb{R}^{N \times N}$ the set of real $N$ by $N$ matrices, equipped with the standard structures. The entries of $A \in \mathbb{R}^{N \times N}$ are denoted by $a_{i j}$, etc.

A matrix $A \in \mathbb{R}^{N \times N}$ is an $M L$-matrix if its off-diagonal entries are nonnegative. For an ML-matrix $A$ its eigenvalue with the largest real part is in fact real (cf., e.g., [3. Thm. 2.1.1 on p. 26], or [22, Section 2.3]), will be called the dominant eigenvalue of $A$, and denoted by $d(A)$. If $A$ has all entries nonnegative then $d(A)$ is equal to the spectral radius of $A$.

$\langle\cdot, \cdot\rangle$ denotes the standard (Euclidean) inner product in $\mathbb{R}^{N} .\|\cdot\|$ will stand, depending on the context, either for the standard Euclidean norm on $\mathbb{R}^{N}$, or for the operator norm on $\mathcal{L}\left(\mathbb{R}^{N}\right)$, the vector space of linear maps from $\mathbb{R}^{N}$ into $\mathbb{R}^{N}$, induced by the standard Euclidean norm.

We denote

$$
\begin{aligned}
\mathbb{R}_{+}^{N} & :=\left\{x=\operatorname{col}\left(x_{1}, \ldots, x_{n}\right): x_{i} \geq 0,1 \leq i \leq n\right\}, \\
\mathbb{R}_{++}^{N} & :=\left\{x=\operatorname{col}\left(x_{1}, \ldots, x_{n}\right): x_{i}>0,1 \leq i \leq n\right\} .
\end{aligned}
$$

Our method consists in finding a sort of Lyapunov function, more precisely, a homogeneous polynomial $V$ of the coordinates $x_{1}, \ldots, x_{N}$ of $x$, taking positive values on $\mathbb{R}_{++}^{N}$, with the property that

- there exists $a>0$ such that

$$
\|x\| \geq a V(x) \text { for all } x \in \mathbb{R}_{++}^{N} ;
$$

- there is a function $b(\cdot)$ defined on the set of ML-matrices, expressed as an elementary function of the entries (in our examples, by means only of 
arithmetic operations, taking square roots and/or maxima/minima), such that for any ML-matrix $A$ the inequality

$$
\langle\nabla V(x), A x\rangle \geq b(A) V(x)
$$

holds for all $x \in \mathbb{R}_{++}^{N}$.

The paper is organized as follows.

In Section 1 the Lyapunov function $V$ is defined as $V(x)=x_{1} \cdot \ldots \cdot x_{N}$. The estimates obtained (Theorem 1.3) are called Kolotilina-type estimates, since their counterparts for nonnegative matrices first appeared in L. Yu. Kolotilina's paper 9 .

In Section 2 the polynomial $V(x)=x_{1}+\ldots+x_{N}$ is used. For time-independent matrices the results obtained there (Theorems 2.2 and 2.3) specialize to well-known Frobenius estimates (see, e.g., [11, 3.1.1], or [18, Chapter II]).

Section 3 gives possible extensions of the results. Section 4 contains concluding remarks.

\section{Kolotilina-type estimates}

1.1. Basic estimate. Throughout the present subsection we assume that $A:(\alpha, \beta)$ $\rightarrow \mathbb{R}^{N \times N}$, where $-\infty \leq \alpha<\beta \leq \infty$, with locally integrable entries.

Consider a system of linear ODEs

$$
x^{\prime}=A(t) x, \quad t \in(\alpha, \beta) .
$$

For any $t_{0} \in(\alpha, \beta)$ and any $x_{0} \in \mathbb{R}^{N}$ there exists a unique solution $[(\alpha, \beta) \ni t \mapsto$ $x\left(t ; t_{0}, x_{0}\right) \in \mathbb{R}^{N}$ ] of (1.1) satisfying the initial condition $x\left(t_{0}\right)=x_{0}$. The solution is understood in the Carathéodory sense: The function $\left[t \mapsto x\left(t ; t_{0}, x_{0}\right)\right]$ is absolutely continuous on any compact subinterval of $(\alpha, \beta), x\left(t_{0} ; t_{0}, x_{0}\right)=x_{0}$, and (1.1) is satisfied for Lebesgue-a.e. $t \in(\alpha, \beta)$.

System (1.1) is said to be cooperative if for each $t \in(\alpha, \beta)$ the matrix $A(t)$ is an ML-matrix.

Proposition 1.1. Assume that (1.1) is cooperative. Then for each $t_{0} \in(\alpha, \beta)$ and each $x_{0} \in \mathbb{R}_{++}^{N}$, if we denote $x\left(t ; t_{0}, x_{0}\right)=\operatorname{col}\left(x_{1}(t), \ldots, x_{N}(t)\right)$, the inequality

$$
\prod_{i} x_{i}(t) \geq \exp \left(\int_{t_{0}}^{t}\left(\operatorname{tr} A(\tau)+2 \sum_{j<k} \sqrt{a_{j k}(\tau) a_{k j}(\tau)}\right) d \tau\right) \prod_{i} x_{i}\left(t_{0}\right)
$$

holds for all $t \in\left(t_{0}, \beta\right)$.

Proof. Since the functions $x_{i}(\cdot), 1 \leq i \leq N$, are a.e. differentiable, we have that a.e. on $\left(t_{0}, \beta\right)$ there holds

$$
\begin{aligned}
\left(\prod_{i} x_{i}\right)^{\prime} & =\sum_{i}\left(x_{i}^{\prime} \prod_{j \neq i} x_{j}\right)=\sum_{i}\left(\left(\sum_{k} a_{i k}(t) x_{k}\right) \prod_{j \neq i} x_{j}\right) \\
& =\sum_{i}\left(a_{i i}(t) x_{i} \prod_{j \neq i} x_{j}\right)+\sum_{i}\left(\left(\sum_{k \neq i} a_{i k}(t) x_{k}\right) \prod_{j \neq i} x_{j}\right) .
\end{aligned}
$$


The first term is equal to

$$
\operatorname{tr} A(t) \prod_{i} x_{i}
$$

Regarding the second term, we have

$$
\sum_{i}\left(\left(\sum_{k \neq i} a_{i k}(t) x_{k}\right) \prod_{j \neq i} x_{j}\right)=\sum_{j<k}\left(\left(a_{j k}(t)\left(x_{k}\right)^{2}+a_{k j}(t)\left(x_{j}\right)^{2}\right) \prod_{\substack{i \neq j \\ i \neq k}} x_{i}\right) .
$$

But, as

$$
a_{j k}(t)\left(x_{k}\right)^{2}+a_{k j}(t)\left(x_{j}\right)^{2} \geq 2 \sqrt{a_{j k}(t) a_{k j}(t)} x_{j} x_{k}
$$

for any $j<k$, we have

$$
\left(\sum_{i} \ln x_{i}\right)^{\prime} \geq \operatorname{tr} A(t)+2 \sum_{j<k} \sqrt{a_{j k}(t) a_{k j}(t)}
$$

a.e. on $\left(t_{0}, \beta\right)$. Keeping in mind that the functions $x_{i}(\cdot)$ are absolutely continuous on compact subintervals, we can integrate the above inequality to get the desired result.

1.2. Monotone linear random dynamical systems. This subsection presents an application of Proposition 1.1 to monotone linear random dynamical systems generated by random cooperative systems of linear ODEs. We start by sketching briefly the construction of such a DS.

Let $(\Omega, \mathfrak{F}, \mathbb{P})$ be a probability space: $\Omega$ is a set, $\mathfrak{F}$ is a $\sigma$-algebra of subsets of $\Omega$, and $\mathbb{P}$ is a probability measure defined for all $F \in \mathfrak{F}$. We assume in addition that the measure $\mathbb{P}$ is complete.

For a metric space $S, \mathfrak{B}(S)$ denotes the $\sigma$-algebra of Borel subsets of $S$.

By a measure-preserving dynamical system on $(\Omega, \mathfrak{F}, \mathbb{P})$ we understand a $(\mathfrak{B}(\mathbb{R}) \otimes$ $\mathfrak{F}, \mathfrak{F}$ )-measurable mapping $\theta: \mathbb{R} \times \Omega \rightarrow \Omega$ satisfying the following (where we write, for $\left.t \in \mathbb{R}, \theta_{t}(\cdot)=\theta(t, \cdot)\right)$ :

$$
\begin{aligned}
& \text { - } \theta_{0}=\operatorname{Id}_{\Omega}, \\
& \text { - } \theta_{s+t}=\theta_{t} \circ \theta_{s}, \text { for any } s, t \in \mathbb{R}, \\
& \text { - } \mathbb{P}\left(\theta_{t}(F)\right)=\mathbb{P}(F) \text {, for any } t \in \mathbb{R} \text { and any } F \in \mathfrak{F} .
\end{aligned}
$$

We denote a measure-preserving $\operatorname{DS}$ as $\left((\Omega, \mathfrak{F}, \mathbb{P}),\left(\theta_{t}\right)_{t \in \mathbb{R}}\right)$. We usually write $\omega \cdot t$ instead of $\theta_{t}(\omega)$.

We always assume that $\left((\Omega, \mathfrak{F}, \mathbb{P}),\left(\theta_{t}\right)_{t \in \mathbb{R}}\right)$ is ergodic: For any invariant $F \in \mathfrak{F}$, either $\mathbb{P}(F)=0$ or $\mathbb{P}(F)=1$.

The first assumption we make on $A$ is:

(A0) $A: \Omega \rightarrow \mathbb{R}^{N \times N}$ belongs to $L_{1}\left((\Omega, \mathfrak{F}, \mathbb{P}), \mathbb{R}^{N \times N}\right)$.

Under (A0) it can be shown (see 1, Ex. 2.2.8]) that for any $\omega \in \Omega$ and any $x_{0} \in \mathbb{R}^{N}$ there exists a unique (Carathéodory) solution $\left[\mathbb{R} \ni t \mapsto x\left(t ; \omega, x_{0}\right) \in \mathbb{R}^{N}\right]$ of the random linear system of ODEs

$$
x^{\prime}=A(\omega \cdot t) x
$$

satisfying the initial condition $x(0)=x_{0}$. Moreover, the mapping

$$
\left[\Omega \times \mathbb{R} \times \mathbb{R}^{N} \ni\left(\omega, t, x_{0}\right) \mapsto x\left(t ; \omega, x_{0}\right) \in \mathbb{R}^{N}\right]
$$

is $\left(\mathfrak{F} \otimes \mathfrak{B}(\mathbb{R}) \otimes \mathfrak{B}\left(\mathbb{R}^{N}\right), \mathfrak{B}\left(\mathbb{R}^{N}\right)\right)$-measurable. 
For $\omega \in \Omega$ and $t \in \mathbb{R}$ define $U_{\omega}(t) \in \mathcal{L}\left(\mathbb{R}^{N}\right)$ by

$$
U_{\omega}(t) x_{0}:=x\left(t ; \omega, x_{0}\right), \quad x_{0} \in \mathbb{R}^{N} .
$$

The family of linear operators $\left\{U_{\omega}(t)\right\}_{\omega \in \Omega, t \in \mathbb{R}}$ has the following properties:

- $U_{\omega}(0)=\operatorname{Id}_{\mathbb{R}^{N}}$, for any $\omega \in \Omega$.

- $U_{\omega}(t+s)=U_{\omega \cdot s}(t) \circ U_{\omega}(s)$, for any $\omega \in \Omega, s, t \in \mathbb{R}$.

We call $\left\{U_{\omega}(t)\right\}_{\omega \in \Omega, t \in \mathbb{R}}$ the linear random dynamical system generated by (1.4).

Proposition 1.2. Assume (A0). Then there exist:

- an invariant $\Omega_{1} \subset \Omega$ with $\mathbb{P}\left(\Omega_{1}\right)=1$, and

- a real number $\lambda$,

with the property that

$$
\lim _{t \rightarrow \infty} \frac{\ln \left\|U_{\omega}(t)\right\|}{t}=\lambda
$$

for each $\omega \in \Omega_{1}$.

Such a $\lambda$ will be referred to as the top Lyapunov exponent for (1.4).

Proof. See, e.g., [1, Thm. 3.3.10].

The next assumption we make is:

(A1) For each $\omega \in \Omega$ the matrix $A(\omega)$ is an $M L$-matrix.

We will call (1.4) for which (A1) is satisfied a cooperative random linear system of ODEs.

An important property of the linear random DS generated by (1.4) satisfying (A0) and (A1) is that it is monotone: $U_{\omega}(t) \mathbb{R}_{+}^{N} \subset \mathbb{R}_{+}^{N}$ and $U_{\omega}(t) \mathbb{R}_{++}^{N} \subset \mathbb{R}_{++}^{N}$, for all $\omega \in \Omega$ and $t \geq 0$ (for a proof see, e.g., [4, Lemma 5.2.1], or [12]).

We are ready now to formulate the main theorem of the paper.

Theorem 1.3 (Kolotilina-type estimate). Under (A0) and (A1),

$$
\lambda \geq \frac{1}{N} \int_{\Omega}\left(\operatorname{tr} A(\omega)+2 \sum_{j<k} \sqrt{a_{j k}(\omega) a_{k j}(\omega)}\right) d \mathbb{P}(\omega) .
$$

Proof. Since $0 \leq \sqrt{a_{j k} a_{k j}} \leq \frac{1}{2}\left(a_{j k}+a_{k j}\right)$, it follows from (A0) that the functions $\sqrt{a_{j k}(\cdot) a_{k j}(\cdot)}$ are in $L_{1}((\Omega, \mathfrak{F}, \mathbb{P}))$, for any $j<k$. An application of the Birkhoff ergodic theorem (see, e.g., [1, Appendix A.1]) yields the existence of $\tilde{\Omega} \subset \Omega, \mathbb{P}(\tilde{\Omega})=$ 1 , such that for each $\omega \in \tilde{\Omega}$ there holds

$$
\begin{aligned}
\lim _{t \rightarrow \infty} \frac{1}{t} \int_{0}^{t}(\operatorname{tr} A(\omega \cdot \tau) & \left.+2 \sum_{j<k} \sqrt{a_{j k}(\omega \cdot \tau) a_{k j}(\omega \cdot \tau)}\right) d \tau \\
& =\int_{\Omega}\left(\operatorname{tr} A(\cdot)+2 \sum_{j<k} \sqrt{a_{j k}(\cdot) a_{k j}(\cdot)}\right) d \mathbb{P}(\cdot) .
\end{aligned}
$$

Fix $\omega \in \tilde{\Omega} \cap \Omega_{1}$, where $\Omega_{1}$ is as in Proposition [1.2, By taking $t_{0}=0, x_{0}=$ $\operatorname{col}(1, \ldots, 1)$ and $x(t)=x\left(t ; t_{0}, x_{0}\right)=x\left(t ; \omega, x_{0}\right)$ in Proposition 1.1 we obtain, after 
straightforward calculation, that

$$
\begin{gathered}
\ln \|x(t)\| \geq \max _{i} \ln x_{i}(t) \geq \frac{1}{N} \sum_{i} \ln x_{i}(t) \\
\geq \frac{1}{N} \int_{0}^{t}\left(\operatorname{tr} A(\omega \cdot \tau)+2 \sum_{j<k} \sqrt{a_{j k}(\omega \cdot \tau) a_{k j}(\omega \cdot \tau)}\right) d \tau
\end{gathered}
$$

for each $t>0$.

Further,

$$
\ln \left\|U_{\omega}(t)\right\| \geq \ln \|x(t)\|-\frac{1}{2} \ln N, \quad t>0 .
$$

Gathering Proposition 1.2, (1.5), (1.6) and (1.7) concludes the proof.

It is good to pause here to explain the reason why we have chosen to put our results in the setting of random DSs. Indeed, this setting covers a lot of cases. For instance, consider a nonautonomous linear ODE system

$$
x^{\prime}=\tilde{A}(t) x
$$

for which the function $\tilde{A}:(-\infty, \infty) \rightarrow \mathbb{R}^{N \times N}\left(\right.$ or $\left.\tilde{A}:[0, \infty) \rightarrow \mathbb{R}^{N \times N}\right)$ is bounded and continuous. (1.8) can be embedded into a family

$$
x^{\prime}=A(\omega \cdot t) x
$$

parameterized by $\omega \in \Omega$, where a compact metrizable space $\Omega$ is the closure, in a suitable topology, of some set whose members are time-translates of (some extension of) $\tilde{A} . \quad \Omega$ is considered with the translation flow on it. For details of a similar construction the interested reader is referred to [16, pp. 81-82]. Now, by the theory presented in [7], extreme values of the exponential growth rates of solutions of (1.8) can be expressed as Lyapunov exponents for some invariant ergodic measures for the translation flow on $\Omega$.

\subsection{Examples.}

Example 1.4. Assume that $A: \mathbb{R} \rightarrow \mathbb{R}^{N \times N}$ is a continuous $T$-periodic matrix function such that $A(t)$ is an ML-matrix for all $t \in \mathbb{R}$.

Then $\Omega=\mathbb{R} /\{k T: k \in \mathbb{Z}\}, \mathbb{P}$ is the normalized Lebesgue measure on $\Omega$, and $\omega \cdot t=\omega+t$ for any $\omega \in \Omega, t \in \mathbb{R}$ (addition is considered modulo $T$ ).

Fix some $\omega \in \Omega\left(\omega=0\right.$, say) and take the Poincaré map $U_{0}(T)$. Let $P$ denote the matrix of $U_{0}(T)$ in the standard basis. $P$ is nonsingular and has, by monotonicity, all entries nonnegative, so its (positive) spectral radius equals its dominant eigenvalue $d(P)$. It follows via Floquet theory (see, e.g., [5, Section 2.2]) that the top Lyapunov exponent of the linear DS generated by the time-periodic cooperative linear ODE system $x^{\prime}=A(t) x$ is equal to $(1 / T) \ln d(P)$. The estimate in Theorem 1.3 takes the form:

$$
\lambda \geq \frac{1}{N T} \int_{0}^{T}\left(\operatorname{tr} A(t)+2 \sum_{j<k} \sqrt{a_{j k}(t) a_{k j}(t)}\right) d t .
$$

Example 1.5. Assume that $A \in \mathbb{R}^{N \times N}$ is an ML-matrix, and consider the autonomous system of cooperative linear ODEs $x^{\prime}=A x$. Then $\Omega$ is a singleton, 
$U(t)=e^{t A}$ for each $t \in \mathbb{R}$, so we recover the following estimate due to L. Yu. Kolotilina (see [9, Corollary 3], cf. also [21]):

Corollary 1.6 (Kolotilina's estimate). Let $A \in \mathbb{R}^{N \times N}$ be an $M L$-matrix. Then its dominant eigenvalue $d(A)$ satisfies

$$
d(A) \geq \frac{1}{N}\left(\operatorname{tr} A+2 \sum_{j<k} \sqrt{a_{j k} a_{k j}}\right) .
$$

\section{Frobenius-type estimates}

In the present section we apply a different Lyapunov function. This approach is especially useful in the case when matrices have a lot of zero off-diagonal entries. We outline only sketches of proofs.

Proposition 2.1. In the assumptions of Subsection 1.1, for each $t_{0} \in(\alpha, \beta)$ and each $x_{0} \in \mathbb{R}_{++}^{N}$, if we denote $x\left(t ; t_{0}, x_{0}\right)=\operatorname{col}\left(x_{1}(t), \ldots, x_{N}(t)\right)$, the inequality

$$
\sum_{i} x_{i}(t) \geq \exp \left(\min _{i} \sum_{j} a_{i j}(t)\right) \sum_{i} x_{i}\left(t_{0}\right)
$$

holds for all $t \in\left(t_{0}, \beta\right)$.

Proof. We observe that

$$
\sum_{i} x_{i}^{\prime}=\sum_{i} \sum_{j} a_{i j}(t) x_{j} \geq\left(\min _{i} \sum_{j} a_{i j}(t)\right) \sum_{i} x_{i}
$$

holds a.e. on $\left(t_{0}, \beta\right)$, and modify the proof of Proposition 1.1 accordingly.

Theorem 2.2 (Frobenius-type estimate, column version). Under (A0) and (A1),

$$
\lambda \geq \int_{\Omega}\left(\min _{i} \sum_{j} a_{i j}(\omega)\right) d \mathbb{P}(\omega) .
$$

Indication of proof. We copy the proof of Theorem 1.3. applying the fact that the sum of the coordinates of a vector $x$ in $\mathbb{R}_{++}^{N}$ equals the $\ell_{1}$-norm of $x$.

By passing to the dual system and noticing that it is generated by a random linear system of ODEs with matrix transposes (see [1, Ch. 5]), we see that the following holds true:

Theorem 2.3 (Frobenius-type estimate, row version). Under (A0) and (A1),

$$
\lambda \geq \int_{\Omega}\left(\min _{j} \sum_{i} a_{i j}(\omega)\right) d \mathbb{P}(\omega) .
$$

Specializing to a time-independent ML-matrix $A$ we recover the well-known (lower) Frobenius estimates (see, e.g., [11]):

$$
d(A) \geq \min _{i} \sum_{j} a_{i j}, \quad d(A) \geq \min _{j} \sum_{i} a_{i j} .
$$




\section{EXTENSIONS}

When some diagonal entries are largely negative, the contribution from them can worsen the estimates considerably. That can be overcome by considering other polynomials, for instance $V(x)=x_{i} x_{j}$ for suitably chosen indices $i \neq j$ such that $a_{i i}(\cdot)$ and $a_{j j}(\cdot)$ are not too largely negative.

Indeed, when starting from $x\left(t_{0}\right)=\operatorname{col}(1, \ldots, 1)$ we obtain

$$
\begin{aligned}
\left(x_{i} x_{j}\right)^{\prime}=x_{i}^{\prime} x_{j}+x_{i} x_{j}^{\prime} & \geq a_{j i}(t)\left(x_{i}\right)^{2}+a_{i j}(t)\left(x_{j}\right)^{2}+\left(a_{i i}(t)+a_{j j}(t)\right) x_{i} x_{j} \\
& \geq\left(a_{i i}(t)+a_{j j}(t)+2 \sqrt{a_{i j}(t) a_{j i}(t)}\right) x_{i} x_{j},
\end{aligned}
$$

and remembering that

$$
\ln \|x(t)\| \geq \max \left(\ln x_{i}(t), \ln x_{j}(t)\right) \geq \frac{1}{2}\left(\ln x_{i}(t)+\ln x_{j}(t)\right)
$$

we finally obtain, proceeding along the lines of the proof of Theorem 1.3 , that

$$
\lambda \geq \int_{\Omega}\left(\frac{1}{2}\left(a_{i i}(\omega)+a_{j j}(\omega)\right)+\sqrt{a_{i j}(\omega) a_{j i}(\omega)}\right) d \mathbb{P}(\omega) .
$$

For a time-independent specialization see [9, Remark 3 on p. 142].

\section{Concluding Remarks}

Remark 4.1. Theorems 1.3, 2.2, 2.3 are of much importance in the case when the so-called generalized exponential separation holds: as shown in [14, Thms. 4.1(3) and 2.4(3)], under additional assumptions on matrices $A(\cdot)$ (too complicated to be written here, specializing to irreducibility in the autonomous case), for $\mathbb{P}$-a.e. $\omega \in \Omega$ the top Lyapunov exponent $\lambda$ is equal to the limit

$$
\lim _{t \rightarrow \infty} \frac{\ln \left\|U_{\omega}(t) x_{0}\right\|}{t}
$$

for each nonzero $x_{0} \in \mathbb{R}_{+}^{N}$ (in that context, $\lambda$ is called the principal Lyapunov exponent for (1.4)).

Remark 4.2. In the discrete-time case analogs of cooperative systems of the form (1.4) are random systems of matrices having all entries nonnegative. Such systems occur, for instance, in Leslie population models.

In 2] the function $V(x)=x_{1} \cdot \ldots \cdot x_{N}$ was used to obtain lower estimates of the principal characteristic exponent in a random Leslie matrix model. Those estimates were expressed in terms of the permanents of matrices.

\section{REFERENCES}

[1] Ludwig Arnold, Random dynamical systems, Springer Monographs in Mathematics, SpringerVerlag, Berlin, 1998. MR1723992 (2000m:37087)

[2] M. Benaïm and S. J. Schreiber, Persistence of structured populations in environmental models, Theor. Popul. Biol. 76 (2009), no. 1, 19-34, DOI 10.1016/j.tpb.2009.03.007. (not covered in MR)

[3] Abraham Berman and Robert J. Plemmons, Nonnegative matrices in the mathematical sciences, Classics in Applied Mathematics, vol. 9, Society for Industrial and Applied Mathematics (SIAM), Philadelphia, PA, 1994. Revised reprint of the 1979 original. MR 1298430 (95e:15013)

[4] Igor Chueshov, Monotone random systems theory and applications, Lecture Notes in Mathematics, vol. 1779, Springer-Verlag, Berlin, 2002. MR1902500 (2003d:37072) 
[5] Miklós Farkas, Periodic motions, Applied Mathematical Sciences, vol. 104, Springer-Verlag, New York, 1994. MR.1299528 (95g:34058)

[6] Barnabas M. Garay and Josef Hofbauer, Robust permanence for ecological differential equations, minimax, and discretizations, SIAM J. Math. Anal. 34 (2003), no. 5, 1007-1039, DOI 10.1137/S0036141001392815. MR2001657 (2004i:37027)

[7] Russell A. Johnson, Kenneth J. Palmer, and George R. Sell, Ergodic properties of linear dynamical systems, SIAM J. Math. Anal. 18 (1987), no. 1, 1-33, DOI 10.1137/0518001. MR 871817 (88a:58112)

[8] Krešimir Josić and Robert Rosenbaum, Unstable solutions of nonautonomous linear differential equations, SIAM Rev. 50 (2008), no. 3, 570-584, DOI 10.1137/060677057. MR2429450 (2009d:34128)

[9] L. Yu. Kolotilina, Lower bounds for the Perron root of a nonnegative matrix, Linear Algebra Appl. 180 (1993), 133-151, DOI 10.1016/0024-3795(93)90528-V. MR1206413 (94b:15016)

[10] Tufail Malik and Hal L. Smith, Does dormancy increase fitness of bacterial populations in time-varying environments?, Bull. Math. Biol. 70 (2008), no. 4, 1140-1162, DOI 10.1007/s11538-008-9294-5. MR2391183 (2009g:92091)

[11] Marvin Marcus and Henryk Minc, A survey of matrix theory and matrix inequalities, Dover Publications Inc., New York, 1992. Reprint of the 1969 edition. MR1215484

[12] Janusz Mierczyński, A simple proof of monotonicity for linear cooperative systems of ODEs, available at arXiv:1304.6562.

[13] Janusz Mierczyński and Sebastian J. Schreiber, Kolmogorov vector fields with robustly permanent subsystems, J. Math. Anal. Appl. 267 (2002), no. 1, 329-337, DOI 10.1006/jmaa.2001.7776. MR.1886831(2003b:37041)

[14] Janusz Mierczyński and Wenxian Shen, Principal Lyapunov exponents and principal Floquet spaces of positive random dynamical systems. II. Finite-dimensional systems, J. Math. Anal. Appl. 404 (2013), no. 2, 438-458, DOI 10.1016/j.jmaa.2013.03.039. MR3045185

[15] Janusz Mierczyński and Wenxian Shen, Persistence in forward nonautonomous competitive systems of parabolic equations, J. Dynam. Differential Equations 23 (2011), no. 3, 551-571, DOI 10.1007/s10884-010-9181-2. MR2836650 (2012j:35172)

[16] Janusz Mierczyński and Wenxian Shen, Spectral theory for forward nonautonomous parabolic equations and applications, Infinite dimensional dynamical systems, Fields Inst. Commun., vol. 64, Springer, New York, 2013, pp. 57-99. MR2986931

[17] Janusz Mierczyński, Wenxian Shen, and Xiao-Qiang Zhao, Uniform persistence for nonautonomous and random parabolic Kolmogorov systems, J. Differential Equations 204 (2004), no. 2, 471-510, DOI 10.1016/j.jde.2004.02.014. MR2085544(2006f:37111)

[18] Henryk Minc, Nonnegative matrices, Wiley-Interscience Series in Discrete Mathematics and Optimization, John Wiley \& Sons Inc., New York, 1988. A Wiley-Interscience Publication. MR932967 (89i:15001)

[19] Paul L. Salceanu, Robust uniform persistence in discrete and continuous nonautonomous systems, J. Math. Anal. Appl. 398 (2013), no. 2, 487-500, DOI 10.1016/j.jmaa.2012.09.005. MR2990074

[20] Sebastian J. Schreiber, Criteria for $C^{r}$ robust permanence, J. Differential Equations 162 (2000), no. 2, 400-426, DOI 10.1006/jdeq.1999.3719. MR1751711(2001e:92012)

[21] Allen J. Schwenk, Tight bounds on the spectral radius of asymmetric nonnegative matrices, Linear Algebra Appl. 75 (1986), 257-265, DOI 10.1016/0024-3795(86)90193-X. MR825411 (87d:15010)

[22] E. Seneta, Non-negative matrices and Markov chains, Springer Series in Statistics, Springer, New York, 2006. Revised reprint of the second (1981) edition [Springer-Verlag, New York; MR0719544]. MR2209438

Institute of Mathematics and Computer Science, WrocŁaw University of Technology, Wybrzeże Wyspiańskiego 27, PL-50-370 Wrocław, Poland

E-mail address: mierczyn@pwr.edu.pl 